\title{
Pharmacological versus genetic inhibition of heme oxygenase-1 - the comparison of metalloporphyrins, shRNA and CRISPR/Cas9 system
}

\author{
Olga Mucha1*, Paulina Podkalicka1*, Maria Czarnek², Anna Biela1, Mateusz Mieczkowski,

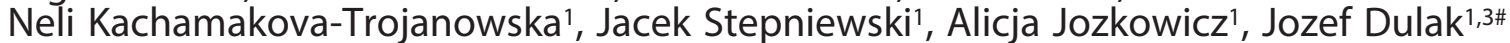 \\ and Agnieszka Loboda1\#囚
}

1Department of Medical Biotechnology, Faculty of Biochemistry, Biophysics and Biotechnology, Jagiellonian University, Kraków, Poland; 2Department of Cell Biochemistry, Faculty of Biochemistry, Biophysics and Biotechnology, Jagiellonian University, Kraków, Poland; ${ }^{3}$ Kardio-Med Silesia, Zabrze, Poland

\begin{abstract}
Inhibition of heme oxygenase-1 (HO-1, encoded by HMOX1), a cytoprotective, anti-apoptotic and anti-inflammatory enzyme, may serve as a valuable therapy in various pathophysiological processes, including tumorigenesis. We compared the effect of chemical inhibitors - metalloporphyrins, with genetic tools - shRNA and CRISPR/ Cas9 systems, to knock-down (KD)/knock-out (KO) HO-1 expression/activity. 293T cells were incubated with metalloporphyrins, tin and zinc protoporphyrins (SnPPIX and ZnPPIX, respectively) or were either transduced with lentiviral vectors encoding different shRNA sequences against HO-1 or were modified by CRISPR/Cas9 system targeting HMOX1. Metalloporphyrins decreased $\mathrm{HO}$ activity but concomitantly strongly induced HO-1 mRNA and protein in 293T cells. On the other hand, only slight basal HO-1 inhibition in shRNA KD 293T cell lines was confirmed on mRNA and protein level with no significant effect on enzyme activity. Nevertheless, silencing effect was much stronger when CRISPR/Cas9-mediated knock-out was performed. Most of the clones harboring mutations within HMOX1 locus did not express $\mathrm{HO}-1$ protein and failed to increase bilirubin concentration after hemin stimulation. Furthermore, CRISPR/Cas9-mediated HO-1 depletion decreased 293T viability, growth, clonogenic potential and increased sensitivity to $\mathrm{H}_{2} \mathrm{O}_{2}$ treatment. In summary, we have shown that not all technologies can be used for inhibition of $\mathrm{HO}$ activity in vitro with the same efficiency. In our hands, the most potent and comprehensible results can be obtained using genetic tools, especially CRISPR/Cas9 approach.
\end{abstract}

Key words: CRISPR/Cas9, shRNA, inhibitors, heme oxygenase-1, HO1, off-target

Received: 08 December, 2017; revised: 19 January, 2018; accepted: 07 March, 2018; available on-line: 25 April, 2018

e-mail: agnieszka.loboda@uj.edu.pl

*equally contributed as the first authors

\#equally contributed as senior authors

Abbreviations: CRISPR, clustered regularly interspaced short palindromic repeats; HO-1, heme oxygenase-1; KD, knock-down; SnPPIX, tin protoporphyrin; ZnPPIX, zinc protoporphyrin

\section{INTRODUCTION}

Heme oxygenase-1 (HO-1, encoded by HMOX1), first characterized in 1968 (Tenhunen et al., 1968), is an enzyme responsible for the degradation of pro-oxidant heme, which leads to the release of carbon monoxide
(CO), ferrous ion $\left(\mathrm{Fe}^{2+}\right)$, and biliverdin. Besides its antioxidant function (Stocker, 1990), HO-1 is considered as a regulator of cellular homeostasis, a modulator of inflammatory response and angiogenesis. Unfortunately, all of potentially beneficial properties of HO-1 can contribute to pathophysiological processes, such as diabetes, hypertension or tumorigenesis (Deshane et al., 2005). Indeed, a growing body of evidence suggests an important role of HO-1 in various types of cancer (Loboda et al., 2015). Therefore, inhibition of HO-1 can potentially serve as an anticancer therapy.

Several approaches for HO-1 silencing are used in experimental settings; however, due to the off-target effects, their experimental and possibly therapeutic application, at least in some cases, is strongly limited. Pharmacological agents, like commonly used metalloporphyrins might, apart from targeting $\mathrm{HO}$, modulate the activity of nitric oxide synthase (NOS), soluble guanylate cyclase (sGC) and cytochrome P450 (Loboda et al., 2015). The most commonly used genetic tools for specific HO-1 inhibition are small interfering RNA (siRNA) or short hairpin RNA (shRNA) that bind to a complementary sequence in targeted mRNA and predominantly induce its cleavage (Rao et al., 2009). In vitro, decreased levels of HO-1 achieved by RNAi resulted in increase in apoptosis of lung cancer cells (Kim et al., 2008) and augmented sensitivity to anticancer therapy in pancreatic cancer cells (Berberat et al., 2005). Moreover, siRNA-mediated HO-1 downregulation led to diminished growth of tumours in vivo in the orthotopic model of hepatocellular carcinoma (Sass et al., 2008). Although the RNAi approach is very useful in experimental settings, its potential therapeutic application remains limited. Major obstacles include inefficient delivery of the genetic material, variable knockdown efficiencies, poor stability due to the degradation by RNases present in body fluids and, in some cases, the possible induction of immune response (Reynolds et al., 2006; Aagaard \& Rossi, 2007). Additionally, siRNA and shRNA sequences can exhibit off-target effects by inhibiting undesired genes (Qiu et al., 2005).

CRISPR/Cas9 system was adopted from bacterial immune defense system against foreign DNA and modified for the purpose of genome engineering. In this approach, Cas9 promotes genome editing by stimulating a double-strand breaks (DSB) formation at a target genomic locus. DSB can be repaired either by non-homologous end joining (NHEJ) or homology-directed repair (HDR). DSB repair by NHEJ often results in small insertions or 
deletions (indels) and, if DSB occurred within coding region of a gene, indel mutations can change the mRNA reading frame leading to the introduction of premature stop codon and, as a consequence, gene knock-out (Hendriks et al., 2016). CRISPR/Cas9 has provided an easy and efficient genome editing tool in cultured human cells (Cho et al., 2013; Cong et al., 2013; Mali et al., 2013), Drosophila (Gratz et al., 2013), zebrafish (Hwang et al., 2013), or plants (Xie \& Yang 2013) and currently is a widely used approach for generation of knock-out animal models, due to its simplicity, efficiency, and specificity (Housden et al., 2017). As a new and still developing method, its advantages, drawbacks, and applications still need to be determined.

The aim of the present study was to compare the effectiveness of both pharmacological and genetic inhibition of HO-1 in a model cell line, human embryonic kidney 293T cells, and to identify the possible benefits and limitations of the used methods.

\section{MATERIALS AND METHODS}

Cell culture. Human embryonic kidney 293T cells (kindly obtained from dr Maciej Wiznerowicz from Wielkopolskie Centrum Onkologii, Poznan, Polska) were cultured under standard conditions $\left(37^{\circ} \mathrm{C}, 5 \% \mathrm{CO}_{2}\right)$ in high glucose (4.5 g/L) Dulbecco's Modified Eagle's medium - DMEM (Lonza, Basel, Switzerland), supplemented with 10\% fetal bovine serum (FBS, Biowest, Nuaillé, France/EURx, Gdansk, Polska) and antibiotics: $100 \mathrm{U} /$ $\mathrm{mL}$ penicillin and $100 \mu \mathrm{g} / \mathrm{mL}$ streptomycin (Lonza, Basel, Switzerland).

Cells stimulation. Cells growing in 96-well plates (3000 or $5000 /$ well), 48-well plates (50000/well), 24well plates (200000/well), 6-well plates (650000/well) or $10 \mathrm{~cm}$ dishes $\left(2.0 \times 10^{6} / \mathrm{dish}\right)$ were stimulated for cell viability or proliferation, immunofluorescence, RNA isolation, protein analysis and $\mathrm{HO}$ activity, respectively. SnPPIX and ZnPPIX (Frontier Scientific, Logan, UT, USA, dissolved in DMSO) were used at $0.5-25 \mu \mathrm{M}$ for $6 \mathrm{~h}$ or $24 \mathrm{~h}$, whereas hemin (Calbiochem, San Diego, CA, USA), was dissolved in phosphate buffer with 10 $\mathrm{mM} \mathrm{NaOH}$ and added at $50 \mu \mathrm{M}$ for $6 \mathrm{~h}$ or at $10 \mu \mathrm{M}$ for $24 \mathrm{~h}$. Hydrogen peroxide $\left(\mathrm{H}_{2} \mathrm{O}_{2}\right)$ at $0.05-0.5 \mathrm{mM}$ concentration was used for $24 \mathrm{~h}$ stimulation. The control cells received the appropriate solvent.

Production of lentiviral vectors encoding shRNA sequences against $\boldsymbol{H} \boldsymbol{M O X 1}$. Four 29-mer shRNA constructs (shHO-1 A-D) against human HMOX1 and one 29-mer non-targeting shRNA (scrambled shRNA) in pGFP-C-shLenti vectors were purchased from OriGene (Rockville, MD, USA). 293T cells were transfected with plasmids of interest and two helper plasmids: pMD2.G and psPAX2as described previously (Szulc et al., 2006) using polyethylenimine - PEI (Polysciences, Inc., Warrington, PA, USA) as a transfection reagent. The plasmids were a gift from Didier Trono (Addgene plasmids \#12259 [pMD2.G] and \#12260 [psPAX2]). Transfection efficiency was monitored under fluorescent microscope by the assessment of GFP positive cells. $48 \mathrm{~h}$ and $72 \mathrm{~h}$ after transfection, media containing lentiviral vectors were collected and pooled. The vector titers were determined by transduction of 293T cells with lentiviral vectors and subsequent analysis of the fraction of GFP positive cells using flow cytometer (BD Fortessa).

Stable transduction with lentiviral vectors. 293T cells were transduced with lentiviral vectors encoding either scrambled shRNA (control cells) or one of four
shRNAs against HMOX1 (shHO-1 A-D) in the presence of $5 \mu \mathrm{g} / \mathrm{mL}$ polybrene (Sigma-Aldrich, St. Louis, MO, USA). Multiplicity of infection (MOI) of 20 was used and after $72 \mathrm{~h}$ the GFP positive cells were sorted using MoFlo XDP cell sorter (Beckman-Coulter).

Knock-out 293T generation. For the generation of HO-1 deficient 293T cells pX330-Pac-Cer plasmid that encode sgRNA targeting human HMOX1 locus or an empty vector (control cells) has been used as described previously (Czarnek \& Bereta, 2017). Cell clones containing mutations in $H M O X 1$ locus (assessed by CELI mismatch detection assay) were used for further analyses.

Colony formation assay. 1000 cells were seeded on 12-well plates and grown for 7 days. Afterwards cells were fixed with cold $\left(-20^{\circ} \mathrm{C}\right), 100 \%$ methanol for 20 minutes on ice and stained with $0.05 \%$ (w/v) crystal violet (BioShop, Burlington, Ontario, Canada) in 20\% methanol for 20 minutes at room temperature. Crystal violet was then completely removed and pictures of the plates were taken using Fusion FX5 XT camera (Vilber).

MTT viability assay. To assess viability of cells, MTT (3-(4,5-dimethylthiazol-2-yl)-2,5-diphenyltetrazolium bromide) (Sigma-Aldrich, St. Louis, MO, USA) assay was used. After $24 \mathrm{~h}$ stimulation with $\mathrm{H}_{2} \mathrm{O}_{2}$, cells were incubated with $1 \mathrm{mg} / \mathrm{mL}$ MT'T in medium for $2 \mathrm{~h}$ at $37^{\circ} \mathrm{C}$. Then, formazan crystals were dissolved in 100 $\mu \mathrm{L} /$ well of lysis buffer consisting of $0.6 \%(\mathrm{v} / \mathrm{v})$ acetic acid and $10 \%(\mathrm{w} / \mathrm{v})$ SDS in DMSO and absorbance at $570 \mathrm{~nm}$ with the reference value of $690 \mathrm{~nm}$ was measured using Infinite M200 microplate reader (Tecan).

Cell proliferation. To determine cell growth over the time, CyQUANT Cell Proliferation Assay (Thermo Fisher Scientific, Waltham MA, USA) was performed according to the manufacturer's instructions.

Heme oxygenase activity assay. Heme oxygenase activity was determined in cell lysates according to the method previously described (Foresti et al., 1997) with slight modification regarding the source of biliverdin reductase (BVR). BVR was produced in BL21(DE3) RIL competent bacteria after transformation using pET24a+:rBVR plasmid with kanamycin resistance. Bacteria were cultured in $\mathrm{LB}$ with $30 \mu \mathrm{g} / \mathrm{mL}$ kanamycin at $37^{\circ} \mathrm{C}$ until optical density (OD600) reached values between 0.6-0.8. Induction of rBVR expression was carried out by addition of $0.1 \mathrm{mM}$ Isopropyl $\beta$-D-1-thiogalactopyranoside (IPTG) for $5 \mathrm{~h}$ at $30^{\circ} \mathrm{C}$. Bacteria pellet was dissolved in buffer containing $20 \mathrm{mM}$ imidazol, $300 \mathrm{mM}$ $\mathrm{NaCl}$ and $20 \mathrm{mM}$ Tris/ $\mathrm{HCl}, \mathrm{pH} 7.4$ in ratio: $1 \mathrm{~g}$ of the bacteria to $5 \mathrm{~mL}$ of the buffer and lysed by sonification. After centrifugation (7500 rpm, 5 minutes), purification of the rBVR was performed by loading the supernatant on a column packed with Ni Sepharose HighPerformance resin. Elution of the rBVR was carried out by using buffer with $500 \mathrm{mM}$ imidazol, $300 \mathrm{mM} \mathrm{NaCl}$ and 20 $\mathrm{mM}$ Tris $/ \mathrm{HCl} \mathrm{pH}$ 7.4. After elution protein was dialyzed for $18 \mathrm{~h}$ at $4^{\circ} \mathrm{C}$ against $300 \mathrm{mM} \mathrm{NaCl}$ and $20 \mathrm{mM}$ Tris/ $\mathrm{HCl}$ pH 7.4 buffer. Subsequently protein was condensed to about $10 \mathrm{mg} / \mathrm{mL}$ concentration, glycerol was added to final $50 \%$ concentration and rBVR was frozen at $-20^{\circ} \mathrm{C}$. $25 \mu \mathrm{g} / \mathrm{ml} \mathrm{BVR}$ was used for HO activity assay.

In brief, after $6 \mathrm{~h}$ stimulation with $50 \mu \mathrm{M}$ hemin cells were scraped in $100 \mathrm{mM}$ phosphate buffer with $2 \mathrm{mM}$ $\mathrm{MgCl}_{2}$. After three cycles of freeze (in liquid nitrogen) and thaw (at $37^{\circ} \mathrm{C}$ ) cell lysate was added to a reaction mixture containing NADPH, glucose-6-phosphate, glucose-6-phosphate dehydrogenase (Sigma-Aldrich, St. Louis, MO, USA), BVR and the substrate hemin. After $1 \mathrm{~h}$ incubation of the above mixture in the dark at $37^{\circ} \mathrm{C}$ the reaction was terminated by the addition of chloro- 
form (POCH, Gliwice, Polska). Followed vigorous vortexing (extraction) and centrifugation (phase separation), the concentration of extracted bilirubin was measured by the difference in absorbance between $464 \mathrm{~nm}$ and 530 $\mathrm{nm}\left(\varepsilon=40 \mathrm{mM}^{-1} \mathrm{~cm}^{-1}\right)$ and calculated as picomole of bilirubin formed per milligram protein per hour.

Quantitative RT-PCR. RNA was isolated from cultured cells using Fenozol (A\&A Biotechnology, Gdynia, Polska) with modified Chomczynski and Sacchi method (Chomczynski \& Sacchi, 1987). RNA concentration and purity were determined using NanoDrop 1000 (Thermo Fisher Scientific, Waltham MA, USA). Reverse transcription was performed with SuperScript polymerase (Invitrogen, Carlsbad, CA, USA) according to instructions supplied by the manufacturer. Obtained cDNA was used as a template for quantitative real-time PCR with SybrGreen Mix (Sigma-Aldrich, St. Louis, MO, USA) and specific primers (HMOX1 F:5'-T'TCTTCACCT'TCCCCAACAT'T-3', R: 5'-CAGCTCCTGCAACTCCTCAAA-3'; HMOX2: F: 5'-GATCGTGGAGGAGGCCAACA-3', R: 5'-TAGAAAGGGCAT'T'TACGCAT-3'; EEF-2 F: 5'-GAGATCCAGTGTCCAGAGCAG-3'; R:5'-CTCGT'TGACGGGCAGATAGG-3'). EEF2 (encoding eukaryotic translation elongation factor 2, EF-2) was used for gene expression normalization. The reaction was performed using a StepOnePlus TM Real-time PCR Systems (Applied Biosystems).

Western blot. Cultured cells were lysed with RIPA buffer and 25-30 $\mu \mathrm{g}$ of protein lysates were loaded on $12 \%$ SDS-PAGE gel followed by electrophoresis and wet transfer of proteins to nitrocellulose membrane (30 V/overnight). Membranes were blocked with 5\% non-fat milk in TBS (Tris-buffered saline, BioShop, Burlington, Ontario, Canada) $+0.1 \%(\mathrm{v} / \mathrm{v})$ Tween-20 (BioShop, Burlington, Ontario, Canada) for $1.5 \mathrm{~h}$ at room temperature. Primary antibodies diluted in blocking solution: rabbit anti-HO-1 (ADI-SPA-894, Enzo Life Science, Farmingdale, NY, USA, 1:500), mouse anti- $\alpha$-tubulin (CP06, Calbiochem, San Diego, CA, USA, 1:1000) were used for overnight incubation at $4^{\circ} \mathrm{C}$. Next day, membranes were washed and incubated with secondary antibodies conjugated with HRP: goat anti-mouse (BD Pharmingen, San Diego, CA, USA, 1:10000) and goat anti-rabbit (Cell Signaling Technology, Danvers, MA, USA, 1:5000) for $1 \mathrm{~h}$ at room temperature. After series of washings, luminescent substrate for HRP activity (Immobilon Chemiluminescent HRP Substrate, Merck Millipore, Billerica, MA, USA) was added for 10 minutes and membranes were manually developed on X-ray film.

Immunofluorescence. Cells were fixed in $4 \%$ paraformaldehyde for 10 minutes, permeabilized with $0.05 \%$ $(\mathrm{v} / \mathrm{v})$ TritonX-100 for 5 minutes and incubated for 30 minutes in $0.25 \%(\mathrm{w} / \mathrm{v})$ glycine in PBS. Afterwards, cells were blocked in 10\% (v/v) goat serum (Sigma-Aldrich, St. Louis, MO, USA) in PBS for $1 \mathrm{~h}$ at room temperature followed by incubation (overnight at $4^{\circ} \mathrm{C}$ ) with primary antibody rabbit anti-HO-1 (ADI-SPA-894, Enzo Life Science, Farmingdale, NY, USA) diluted 1:200 in $1 \%$ (v/v) goat serum in PBS. Next day, cells were incubated with secondary antibody goat anti-rabbit Alexa Fluor 488 or 568 (Thermo Fisher Scientific, Waltham MA, USA) diluted $1: 500$ in $1 \%(\mathrm{v} / \mathrm{v})$ goat serum together with $10 \mu \mathrm{g} / \mathrm{mL}$ Hoechst (Sigma-Aldrich, St. Louis, MO, USA) for $2 \mathrm{~h}$ at room temperature, visualized
A

HO activity assay

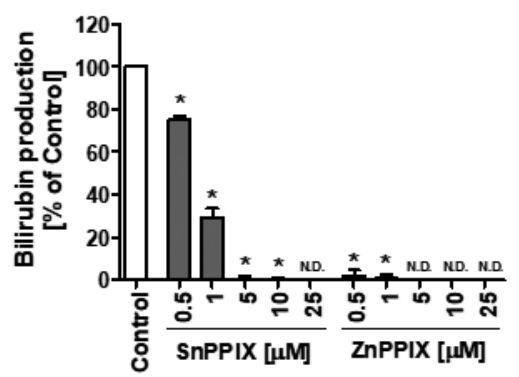

B HMOX1 MRNA 6h

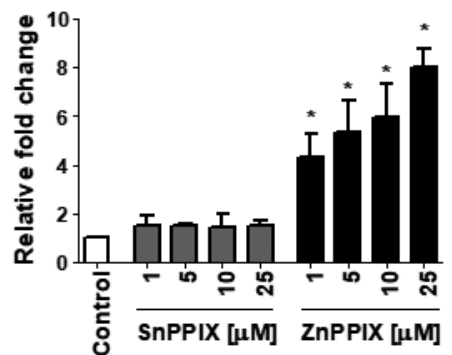

C

HMOX1 MRNA 24h
D

HMOX2 MRNA 6h

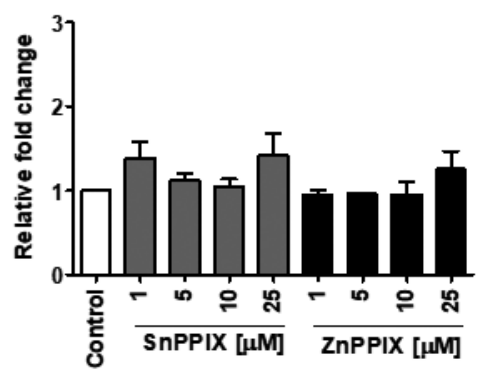

$\mathbf{E}$

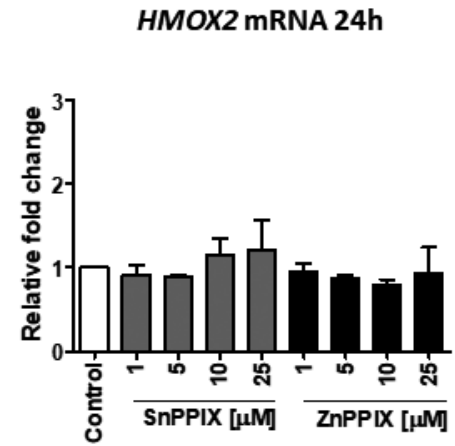

Figure 1. Inhibition of HO activity using SnPPIX and ZnPPIX affects HMOX1 but not HMOX2 mRNA level in 293T cell line.

$\mathrm{HO}$ activity after $6 \mathrm{~h}$ stimulation with SnPPIX/ZnPPIX shown as the percentage of control (mean \pm SD) (A). HMOX1 and HMOX2 mRNA after $6 \mathrm{~h}$ (B, D, respectively) and after $24 \mathrm{~h}$ (C, E, respectively) stimulation with 1-25 $\mu \mathrm{M}$ SnPPIX/ZnPPIX calculated as relative fold change vs. control (mean \pm S.D.). 
under fluorescent microscope and analyzed using ImageJ program.

Statistical analysis. All experiments were performed in duplicates or triplicates and were repeated two or three times. Results are presented as a mean \pm S.D. (standard deviation). Statistical analysis was performed in GraphPad Prism Software using Student's $t$-test or oneway ANOVA. Results were considered statistically significant at $p<0.05$

\section{RESULTS}

\section{Protoporphyrins inhibit $\mathrm{HO}$ activity but upregulate HO-1 mRNA and protein}

Protoporphyrins, SnPPIX and ZnPPIX are widely used inhibitors of $\mathrm{HO}$ activity. In our hands, $6 \mathrm{~h}$ stimulation with either inhibitor led to potent decrease in bilirubin production by $293 \mathrm{~T}$ cells, with much stronger effect of ZnPPIX (Fig. 1A). Nonetheless, already $6 \mathrm{~h}$ stimulation with ZnPPIX resulted in significant induction of HMOX1 mRNA (Fig. 1B). After longer time $(24 \mathrm{~h})$, the stimulatory effect of SnPPIX on HMOX1 expression was also visible (Fig. 1C), but was not as pronounced as after ZnPPIX treatment which was even further increased in comparison to $6 \mathrm{~h}$ stimulation (Fig. 1C). Noteworthy, $6 \mathrm{~h}$ (Fig. 1D) and $24 \mathrm{~h}$ (Fig. 1E) treatment with protoporphyrins had no significant effect on the mRNA expression of constitutive form of $\mathrm{HO}$, HMOX2. The induction of HO-1 protein by protoporphyrins was confirmed by Western blot (Fig. 2A) and immunofluorescent staining (Fig. 2B).

\section{shHO-1 fail to abolish $\mathrm{HO}$ activity induction upon hemin treatment}

293T cells were transduced with lentiviral vectors encoding shRNA against HO-1 (shHO-1 A-D) and scrambled shRNA (further referred to as control cells) and the efficiency of the transduction was monitored using fluorescent microscope and flow cytometer (Fig. 3A). After sorting $\mathrm{GFP}^{+}$cells, HO-1 expression was evaluated. We observed a decrease in basal HMOX1 mRNA in cells transduced with three out of four tested shRNAs (sequences B-D, Fig. 3B); however, $6 \mathrm{~h}$ stimulation with hemin, HO-1 inducer, caused potent induction of HMOX1 in cells transduced either with scrambled shRNA or all shHO-1 (Fig. 3C). HMOX2 mRNA was affected neither by shHO-1 transduction (Fig. 3D) nor by hemin treatment (Fig. 3E). Moreover, basal HO-1 protein level was decreased in cells transduced with shHO-1 B-D sequences (Fig. 4A, B). Hemin-induced HO-1 upregulation was observed in shHO-1 KD cells but it was not as potent as in control cells, as shown by immunofluorescent staining (Fig. 4A) and Western blot (Fig. 4B). Finally, HO activity was not significantly changed in basal conditions (Fig. 4C). In accordance with the mRNA and protein results after hemin stimulation, all shHO1 cells showed increase in $\mathrm{HO}$ activity similar to cells transduced with scrambled shRNA (Fig. 4D).

\section{CRISPR/Cas9-mediated gene editing of HMOX1 locus results in HO-1 deficiency}

In order to obtain HO-1 knock-out cells, 293T cells were transiently transfected with vectors coding for two distinct sgRNAs that target second exon of human HMOX1 locus (Fig. 5A). We chose second exon because it encodes histidine 25 , which is prerequisite for HO-1 catalytic activity and plasmids may be used in future to create mutant clones with catalytically inactive HO-1 instead of HMOX1 knock-out clones. Upon transfection, the cells were selected with puromycin for $24 \mathrm{~h}$ and seeded on 96-well plate to obtain single cell-derived clones. After further amplification of cells, DNA was isolated and CELI mismatch detection assay was performed to evaluate the presence of mutations in HMOX1 amongst the obtained clones (Fig. 5B). Of

A
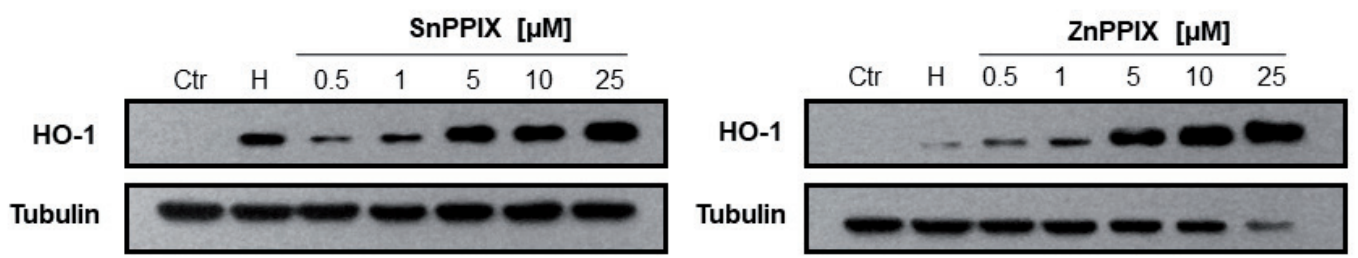

B

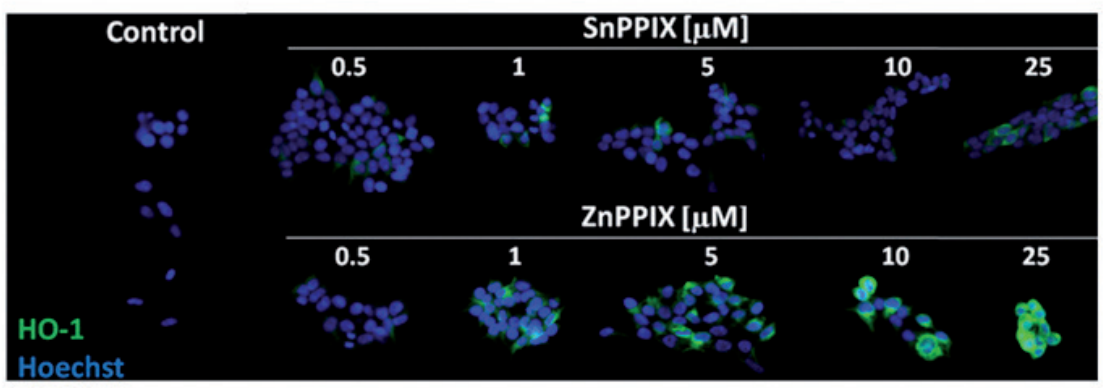

Figure 2. Pharmacological inhibitors of HO activity potently increase HO-1 protein level.

Western blot analysis of HO-1 protein after $24 \mathrm{~h}$ stimulation with inhibitors $(0.05-25 \mu \mathrm{M})$ and $10 \mu \mathrm{M}$ hemin $(\mathrm{H})$ (A). Immunofluorescent detection of HO-1 protein (green) after $24 \mathrm{~h}$ stimulation with protoporphyrins $(0.05-25 \mu \mathrm{M})$, counterstained with Hoechst (B). ${ }^{*} p<0.05$ vs. control. 
A

Transduction efficiency

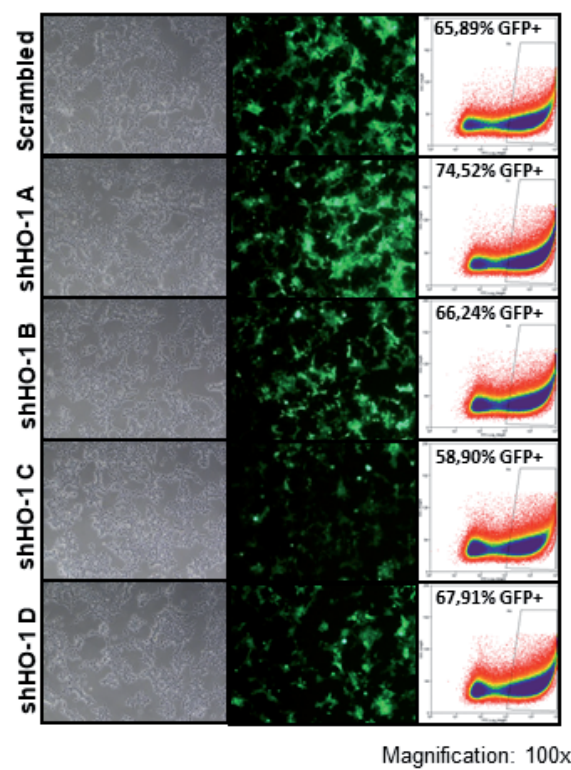

B
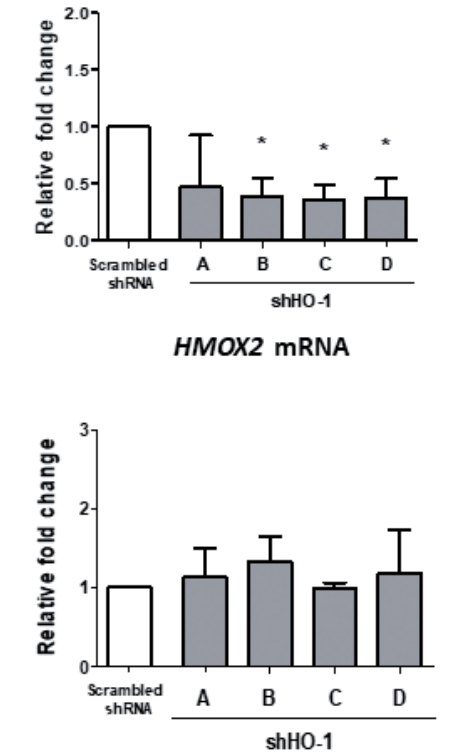

C

HMOX1 mRNA
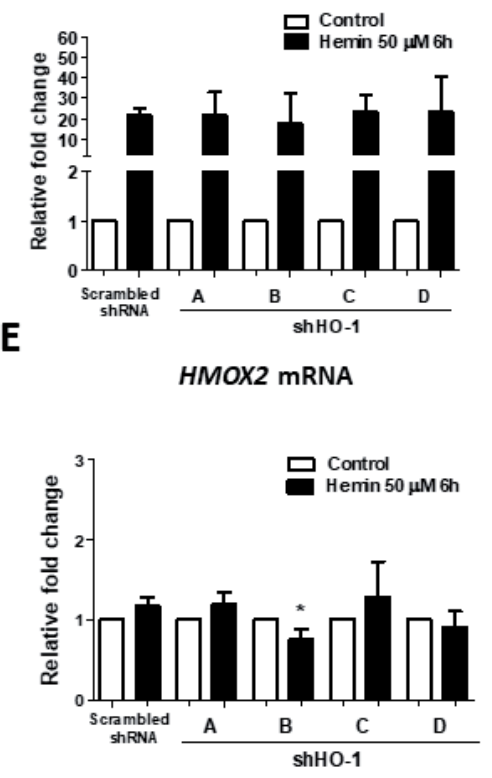

Figure 3. shRNA-mediated HMOX1 silencing fails to abolish HMOX1 induction after hemin treatment.

Representative pictures of cells transduced with lentiviral vectors encoding shRNA against HMOX1 (100x) and percentage of GFP+ cells (A). HMOX1 and HMOX2 (B, D, respectively) expression after transduction with shHO-1 A-D and scrambled shRNA, presented as relative fold change vs. control (mean \pm S.D.).

note, among 31 tested clones, 18 harbored mutations in HMOX1 locus (Fig. 5C).

Due to CRISPR/Cas9-mediated introduction of premature stop codons and subsequent degradation of defective transcripts in nonsense-mediated decay process, we observed diminished level of $H M O X 1$ transcript in several clones harboring mutations in HMOX1 locus. The most pronounced difference was detected in clones 4.4, 4.8 and to a lesser extent in clones 4.14 and 4.20 (Fig. 6A). Hemin-induced HMOX1 expression was
A

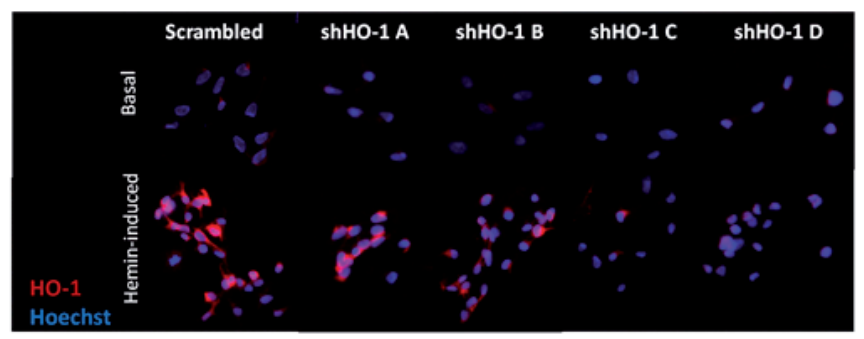

B

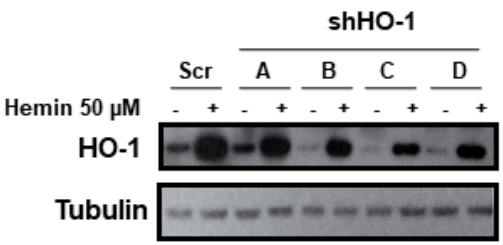

C

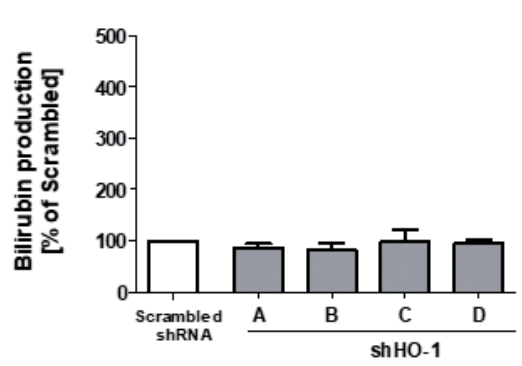

D

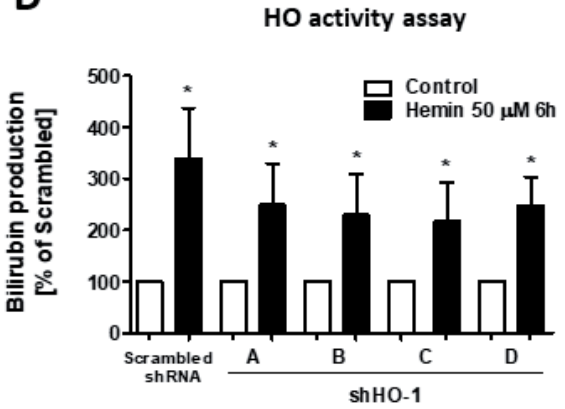

Figure 4. shRNA-mediated silencing of HMOX1 partially decreases basal and hemin-induced protein level, with no effect on HO-1 activity level.

HO-1 protein level assessed in basal and hemin-induced ( $6 \mathrm{~h}$ stimulation) conditions by immunofluorescent staining (red) counterstained with Hoechst (A) and by Western blot (B). HO activity under basal (C) and hemin-stimulated (6 h) conditions (D). HO activity under basal conditions is shown as percentage of control and at hemin-induced conditions as percentage of basal conditions for appropriate cell line (mean \pm S.D.). ${ }^{*} p<0.05$ vs. control. 
A

human HMOX1 locus (13.38 kb)

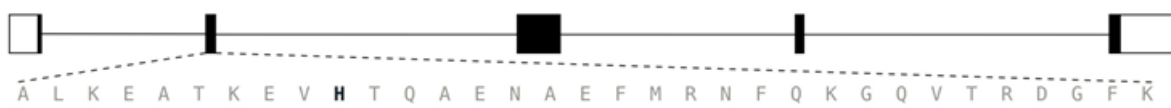

GCCCTGAAGGAGGCCACCAAGGAGGTGCACACCCAGGCAGAGAATGCTGAGTTCATGAGGAACTTTCAGAAGGGCCAGGTGACCCGAGACGGCTTCAAG

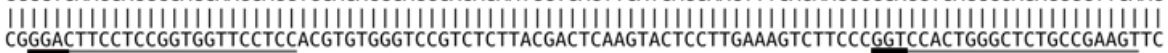

sgRNA 21

sgRNA 4

B

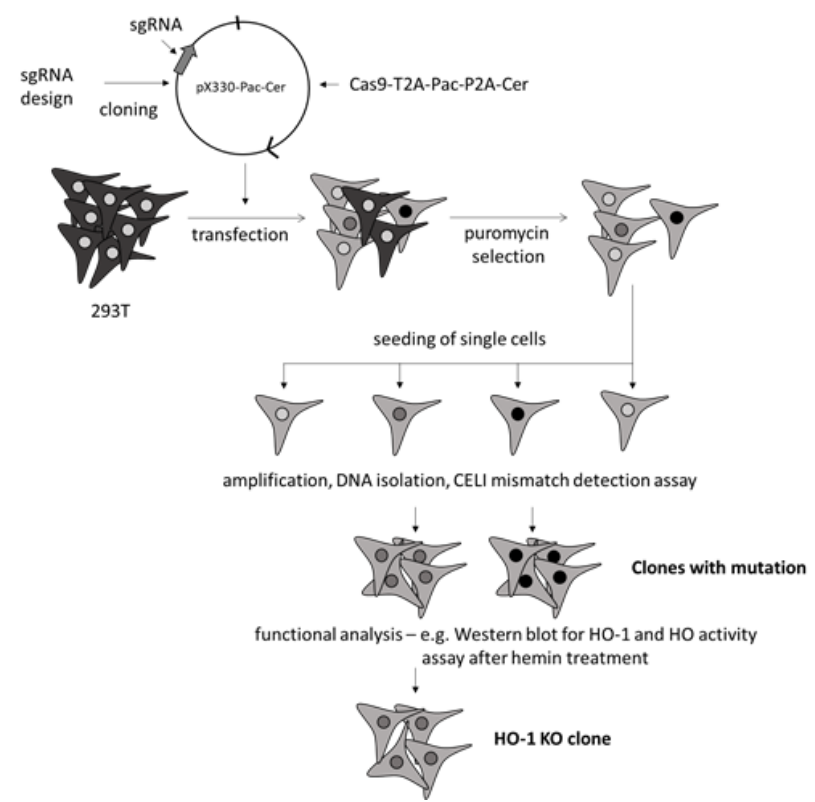

C

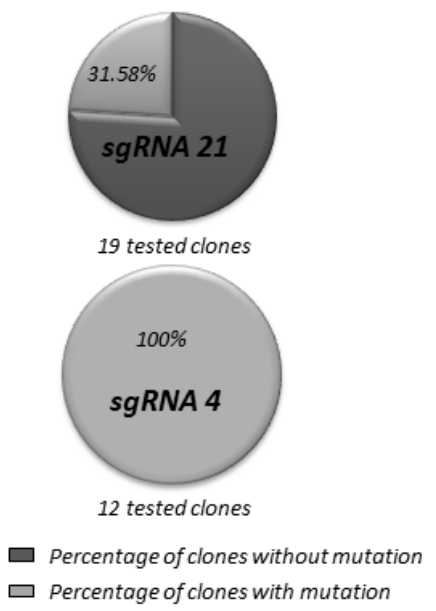

Figure 5. Efficient CRISPR/Cas9-mediated introduction of mutations in HMOX1 in 293T cell line.

Schematic representation of human HMOX1 locus showing the location of HMOX1-targeting sgRNAs (grey lines; PAM sequences are shown in black). Black lines represent introns and rectangles represent exons. DNA sequence of fragment of exon 2 is shown below; sequence of the protein translated from this fragment is given immediately above the nucleotide sequence; $\mathrm{H} 25$ is shown in bold (A). Designed sgRNAs were further utilized to generate HO-1 knockout clones of 293T cells according to general scheme of CRISPR/Cas9mediated gene editing (B). Efficient introduction of mutations in HMOX1 in derived clones was confirmed using CELI mismatch detection assay (C). The results are presented as percentage of clones with detected mutations upon utilization of two sgRNAs sequences.

blunted in several clones, in comparison to hemin-stimulated control cells (Fig. 6B). Basal HMOX2 mRNA level was not affected in majority of clones (Fig. 6C) whereas some inhibition was detected in hemin-stimulated cells (Fig. 6D). Both basal and hemin-induced HO-1 protein level was potently decreased mostly in clones 4.4, 4.8, 4.10 and 4.14 (Fig. 7A, 7B). Most probably, those clones harbor frameshift mutation in all HMOX1 loci. HO activity, which is a combined activity of HO-1 and HO-2, was not changed in basal conditions (Fig. 7C) (except for the clone 4.8), but most importantly, lack of response to hemin stimulation (as the result of blocked HO-1 activity) in all clones was observed (Fig. 7D).

\section{CRISPR/Cas9-mediated HO-1 depletion affects 293T cells viability and growth}

HO-1 depletion in 293T cells caused slight, but significant drop in the cell growth in clones 4.8, 4.10, 4.14, as checked by CyQUANT analysis (Fig. 8A). Furthermore, tested clones (4.8 and 4.10) exhibited decreased viability in basal conditions and significantly higher susceptibility to $0.5 \mathrm{mM} \mathrm{H}_{2} \mathrm{O}_{2}$ stimulation in comparison to control cells (Fig. 8B). Additionally, clonogenic potential of $293 \mathrm{~T}$ cell line was significantly impaired by HO-1 depletion in comparison to control cells (Fig. 8C).

\section{DISCUSSION}

Due to the growing body of evidence suggesting the relevance of HO-1 inhibition as a possible therapeutic strategy, especially in tumour biology, we decided to provide a comprehensive comparison of pharmacological and genetic approaches of HO-1 silencing in a model cell line, human embryonic kidney cells 293T.

Experimental and medical application of metalloporphyrins is limited mostly due to their poor specificity, low bioavailability, and the modulation of pathways not directly related to HO (Grundemar \& Ny, 1997; Schulz et al., 2012). Accordingly, ZnPPIX has been demonstrated to regulate the indoleamine-2,3-dioxygenases activity (Metz et al., 2010), cyclin D1 expression (Rahman et al., 2009), and Wnt/ $\beta$-catenin pathway (Wang et al., 2013). We have previously shown that SnPPIX and ZnPPIX may oppositely regulate the activity of inducible nitric oxide synthase (iNOS), as SnPPIX augmented whereas ZnPPIX decreased production of NO in vascular smooth muscle cells as well as in macrophages and those effects were HO-1 independent (Jozkowicz \& Dulak 2003).

As reported in the present study, both SnPPIX and ZnPPIX almost completely abolished total HO activity, emphasizing their unspecificity towards $\mathrm{HO}$ isoforms. At the same time, we observed strong upregulation of HO-1 both at mRNA and protein levels. This effect was 
A

HMOX1 MRNA

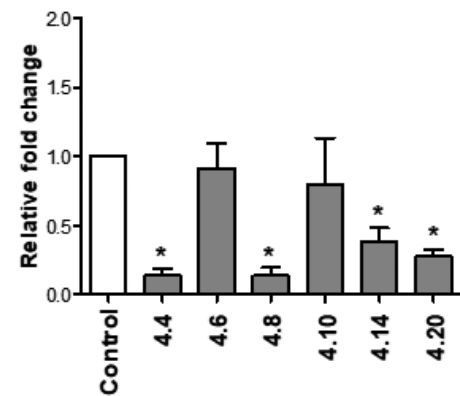

C

HMOX2 MRNA

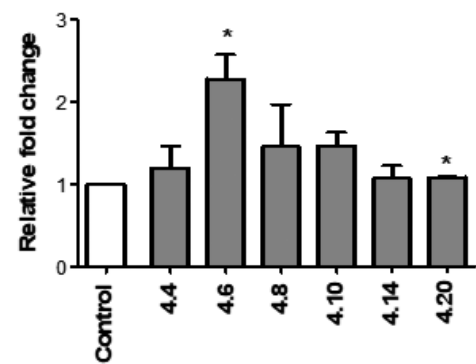

B

HMOX1 MRNA

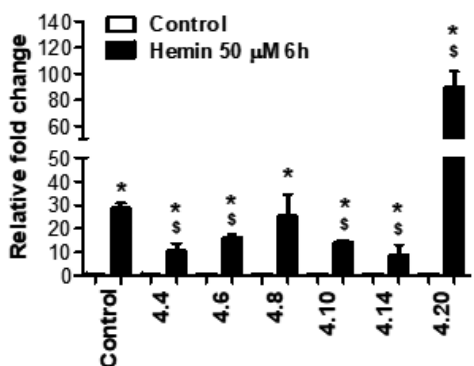

HMOX2 MRNA

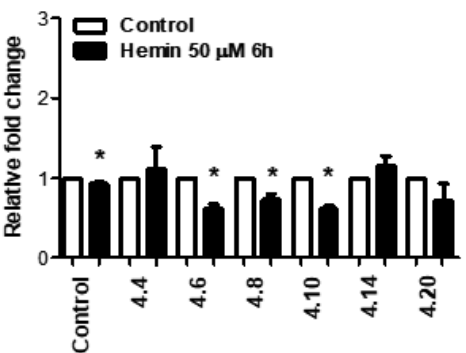

Figure 6. Genetic inhibition of HMOX1 in 293T using CRISPR/Cas9 system significantly decreases HMOX1 but not HMOX2 mRNA level both under basal and hemin-induced conditions.

HMOX1 and HMOX2 expression in control clone and in clones after CRISPR/Cas9-mediated HO-1 excision in basal (A, C, respectively) and in hemin-induced conditions (B, D, respectively). Basal level was calculated as relative fold change vs. control whereas hemin-induced HMOX1 expression is shown as relative fold change vs. basal conditions for each clone (mean \pm S.D.).

also reported in other cell types and tissues (Loboda et al., 2015). It has been proposed that such a dual action might be related to the fact that metalloporphyrins, acting as the analogues of heme, could remove transcrip- tional Bach1 repressor, induce $H M O X 1$ transcription through Nrf2 activation, and simultaneously inhibit its activity by binding to the catalytic pocket of HO-1 (Ogawa et al., 2001). Additionally, it was reported that
A

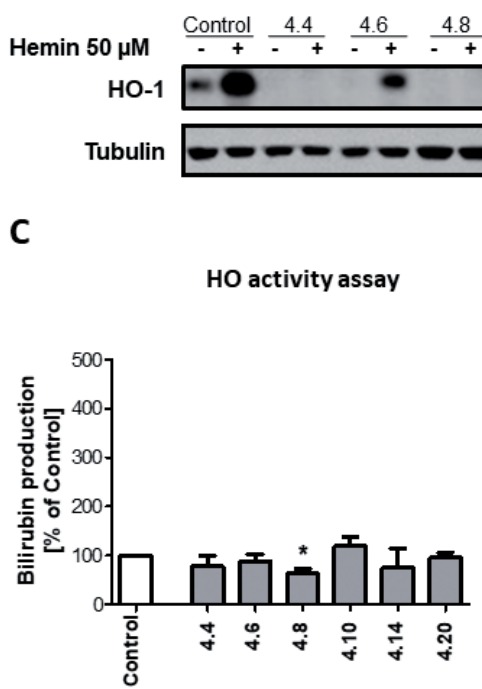

B

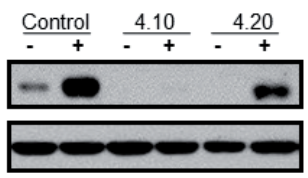

D

HO activity assay
B

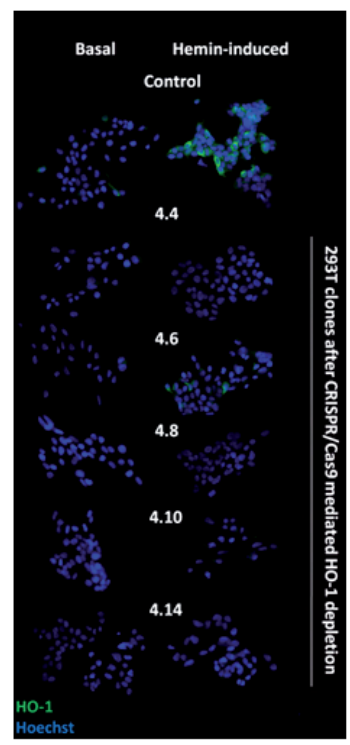

Figure 7. Genetic inhibition of HMOX1 in 293T using CRISPR/Cas9 system leads to potent decrease in HO-1 protein level both under basal and hemin-induced conditions as well as on activity level after treatment with hemin.

Inhibition of HO-1 protein level by CRISPR/Cas9-mediated HO-1 excision checked by immunofluorescent staining (A) and Western Blot (B) both under basal conditions and after $6 \mathrm{~h}$ stimulation with $50 \mu \mathrm{M}$ hemin. $\mathrm{HO}$ activity in basal conditions (C) and after $6 \mathrm{~h}$ stimulation with $50 \mu \mathrm{M}$ hemin (D). HO activity at basal conditions shown as percentage of control and hemin treatment as percentage of basal conditions for appropriate clone (mean \pm S.D.).* $p<0.05$ vs. control; ${ }^{\$} p<0.05$ vs. control cells treated with hemin. 
A

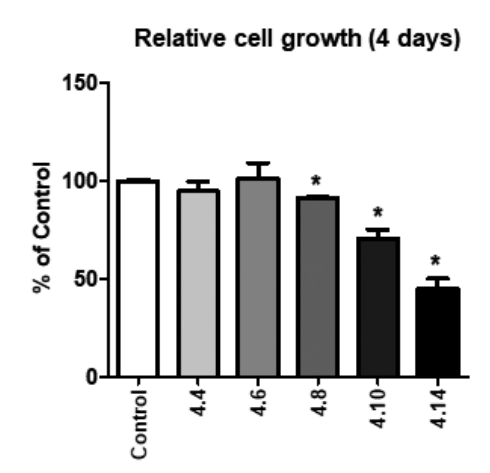

B

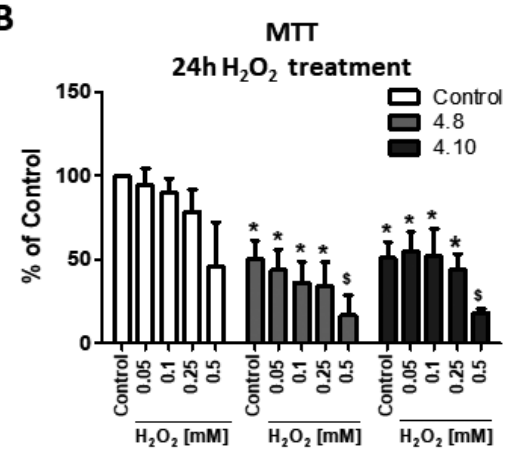

C

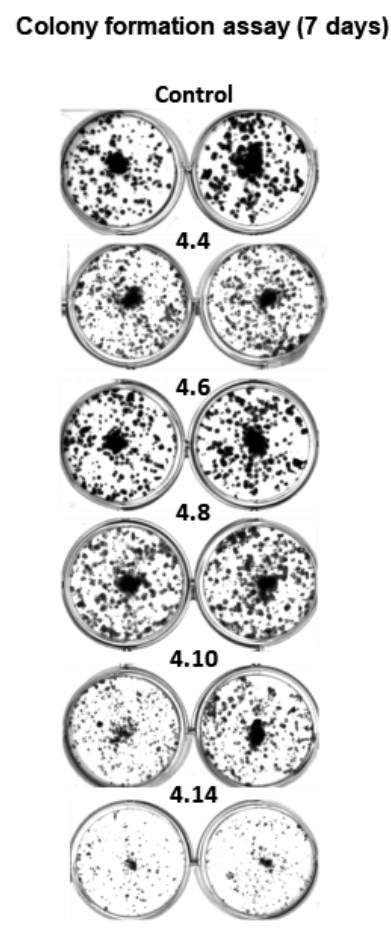

Figure 8. The effect of HMOX1 inhibition by CRISPR/Cas9 system on viability, proliferation and clonogenic potential of $293 \mathrm{~T}$ cell line. CyQUANT assay to estimate relative growth rate was performed after 4 days from seeding. The results are presented as percentage of control (mean \pm S.D.) (A). MTT analysis on cells stimulated with $\mathrm{H}_{2} \mathrm{O}_{2}(0.05-0.5 \mathrm{mM})$ for $24 \mathrm{~h}$ and calculated as percentage of control (mean \pm S.D.) (B). Clonogenic potential checked 7 days after seeding - representative pictures (C). ${ }^{*} p<0.05$ vs. control; ${ }_{p<0.05}$ vs. control cells of each clone.

ZnPPIX mediates binding of early growth response-1 (Egr-1) to the promoter of HO-1, which then induces its expression (Yang et al., 2001). Summarizing, due to HOindependent, unspecific effects, results obtained using metalloporphyrins should be analysed with caution and the involvement of the HO-1 pathway should be always confirmed by more specific methods.

Despite high transduction efficiency in 293T cells obtained in the shRNA approach, most of the tested shRNA sequences decreased HMOX1 mRNA level only to about $35 \%$ of that in control. This partial effect can be cell type-specific but also connected to some internal protective mechanism in cells. The increase in MOI of used vectors would be one option to augment the effectiveness; however, it might also lead to toxicity and off-target effects. Moreover, it appeared important to test few different shRNA sequences, since the efficacy of each of them can vary (Alaoui-Jamali et al., 2009). It was also clearly visible in our hands that shHO-1 sequence A only slightly diminished HMOX1 expression with no changes on protein level. Fortunately, none of tested sequences influenced HMOX2 transcript, which suggests specificity regarding $\mathrm{HO}$ isoforms. This also explains the lack of the effect on basal $\mathrm{HO}$ activity, which probably reflects mostly the activity of HO-2, the isoform predominant in physiological conditions, rather than HO-1. Furthermore, significant induction of HMOX1 $\mathrm{mRNA}$, protein, and activity in response to hemin stimulation was still visible and can be related to insufficient inhibition of newly transcribed HMOX1 mRNA by shRNA. The lack of possibility to inhibit the increase in $\mathrm{HO}$ activity in response to the stimuli is an important and major drawback of this ap- proach as HO-1 is easily-inducible enzyme (Ferrandiz \& Devesa 2008).

In the present study we have clearly shown the advantage of CRISPR/Cas9 technology over shRNA method. Although we observed differences in efficiency of HO-1 depletion between tested clones which can be related to natural variability between cells or some pitfalls of CRISPR/Cas9 technology (Peng et al., 2016) and emphasize the need for careful selection of clones used for further studies, our data clearly demonstrate the usefulness of this method. It has to be stressed that despite the fact that HMOX1 transcript which is most probably mutated, can be still present in cell clones transfected with CRISPR/Cas9 targeting HMOX1, the functionality of the protein is lost. HO-1 protein level in CRISPR/Cas9derived cell clones was diminished, both in basal and hemin-induced conditions and, what is most important, no increase in $\mathrm{HO}$ activity upon stimulation with potent inducer of HO-1, hemin, was observed. This reflects the necessity of validation of the obtained results not only at mRNA or protein levels, but essentially based on the biological functionality, which in our case is a real indicator of the effectiveness of the CRISPR/Cas9 approach.

Importantly, the lack of HO-1 in 293T cells exerts serious biological consequences, including decreased clonogenic potential and cell proliferation as well as diminished cell viability. Moreover, cells with HO-1 deficiency are more susceptible to $\mathrm{H}_{2} \mathrm{O}_{2}$ stimulation, which is connected to the cytoprotective role of HO-1 against stressful conditions. Therefore, HO-1 may be a good anticancer target. Indeed, a growing body of evidence suggests the emergent role of $\mathrm{HO}-1$ in various types of cancer, including melanoma, renal cell carcinoma, pancreatic can- 
cer, and many others. Elevated levels of HO-1 in cancer cells in comparison to cells from healthy tissues has been associated with different aspects of tumour biology, leading to progression of the disease (Loboda et al., 2015). Furthermore, expression of HO-1 can be induced upon anticancer treatment, which often results in decreased effectiveness. Nonetheless, the exact role of HO-1 in tumour biology is multifaceted, since the opposite, anticancer activity of HO-1 was described, for example, in the case of breast or prostate cancer. Thus, although HO-1 might be considered as a therapeutic target, it has to be taken into account that inhibition of HO-1 not in every cancer cell type will be desired. Even more complicated is the fact that contradictory results were obtained within the same type of cancer, which might be at least partially related to different experimental settings. Especially in the case of metalloporphyrins, caution has to be taken in the interpretation of obtained results, since they might not be directly related to the activity of HO-1. Moreover, when considering the shRNA approach, off-target effects may result in the misinterpretation of observed results (Loboda et al., 2015). Thus, we do believe that CRISPR/Cas9 technology may represent the most reliable tool to study specific gene function. Moreover, to our best knowledge, depletion of HO-1 by CRISPR/ Cas9 technology has not been studied so far, and here we report that it can provide an alternative method to study biological effect of HO-1 inhibition in many areas of research, including tumour biology, which is now extensively studied by our group.

\section{Conflict of interest statement}

The authors declare that there is no conflict of interests.

\section{Acknowledgements}

Szymon Czauderna is acknowledged for help in design of CRISPR/Cas9 components.

\section{Acknowledgements of Financial Support}

This work was supported by the grants from the National Science Centre (2016/21/B/NZ1/00293 and 2014/14/M/NZ1/00010) (AL and JD, respectively) and National Centre for Research and Development (PBS2/ B7/15/2014). Faculty of Biochemistry, Biophysics and Biotechnology of Jagiellonian University is a partner of the Leading National Research Center (KNOW) supported by the Ministry of Science and Higher Education.

\section{REFERENCES}

Aagaard L, Rossi JJ (2007) RNAi therapeutics: principles, prospects and challenges. Adv Drug Deliv Rev 59: 75-86. doi: 10.1016/j. addr.2007.03.005

Alaoui-Jamali MA, Bismar TA, Gupta A, Szarek WA, Su J, Song W, Xu Y, Xu B, Liu G, Vlahakis JZ, Roman G, Jiao J, Schipper HM (2009) A novel experimental heme oxygenase-1-targeted therapy for hormone-refractory prostate cancer. Cancer Res 69: 8017-8024. doi: 10.1158/0008-5472.CAN-09-0419

Berberat PO, Dambrauskas Z, Gulbinas A, Giese T, Giese N, Kunzli B, Autschbach F, Meuer S, Buchler MW, Friess H (2005) Inhibition of heme oxygenase-1 increases responsiveness of pancreatic cancer cells to anticancer treatment. Clin Cancer Res 11: 3790-3798. doi: 10.1158/1078-0432.CCR-04-2159

Cho SW, Kim S, Kim JM, Kim JS (2013) Targeted genome engineering in human cells with the Cas9 RNA-guided endonuclease. Nat Biotechnol 31: 230-232. doi: 10.1038/nbt.2507

Chomczynski P, Sacchi N (1987) Single-step method of RNA isolation by acid guanidinium thiocyanate-phenol-chloroform extraction. Anal Biochem 162: 156-159. doi: 10.1006/abio.1987.9999
Cong L, Ran FA, Cox D, Lin S, Barretto R, Habib N, Hsu PD, Wu X, Jiang W, Marraffini LA, Zhang F (2013) Multiplex genome engineering using CRISPR/Cas systems. Science 339: 819-823. doi: 10.1126 / science. 1231143

Czarnek M, Bereta J (2017) SmartFlares fail to reflect their target transcripts levels. Sci Rep 7: 11682. doi: 10.1038/s41598-017-11067-6

Deshane J, Wright M, Agarwal A (2005) Heme oxygenase-1 expression in disease states. Acta Biochim Pol 52: 273-284

Ferrandiz ML, Devesa I (2008) Inducers of heme oxygenase-1. Curr Pharm Des 14: 473-486

Foresti R, Clark JE, Green CJ, Motterlini R (1997) Thiol compounds interact with nitric oxide in regulating heme oxygenase-1 induction in endothelial cells. Involvement of superoxide and peroxynitrite anions. J Biol Chem 272: 18411-18417

Gratz SJ, Cummings AM, Nguyen JN, Hamm DC, Donohue LK, Harrison MM, Wildonger J, O'Connor-Giles KM (2013) Genome engineering of Drosophila with the CRISPR RNA-guided Cas9 nuclease. Genetics 194: 1029-1035. doi: 10.1534/genetics.113.152710

Grundemar L, Ny L (1997) Pitfalls using metalloporphyrins in carbon monoxide research. Trends Pharmacol Sci 18: 193-195

Hendriks WT, Warren CR, Cowan CA (2016) Genome Editing in Human Pluripotent Stem Cells: Approaches, Pitfalls, and Solutions. Cell Stem Cell 18: 53-65. doi: 10.1016/j.stem.2015.12.002

Housden BE, Muhar M, Gemberling M, Gersbach CA, Stainier DY, Seydoux G, Mohr SE, Zuber J, Perrimon N (2017) Loss-of-function genetic tools for animal models: cross-species and cross-platform differences. Nat Rev Genet 18: 24-40. doi: 10.1038/nrg.2016.118

Hwang WY, Fu Y, Reyon D, Maeder ML, Tsai SQ, Sander JD, Peterson RT, Yeh JR, Joung JK (2013) Efficient genome editing in zebrafish using a CRISPR-Cas system. Nat Biotechnol 31: 227-229. doi: $10.1038 / \mathrm{nbt} .2501$

Jozkowicz A, Dulak J (2003) Effects of protoporphyrins on production of nitric oxide and expression of vascular endothelial growth factor in vascular smooth muscle cells and macrophages. Acta Biochim Pol 50: 69-79. doi: 035001069

Kim HR, Kim S, Kim EJ, Park JH, Yang SH, Jeong ET, Park C, Youn MJ, So HS, Park R (2008) Suppression of Nrf2-driven heme oxygenase-1 enhances the chemosensitivity of lung cancer A549 cells toward cisplatin. Lung Cancer 60: 47-56. doi: 10.1016/j.lungcan.2007.09.021

Loboda A, Jozkowicz A, Dulak J (2015) HO-1/CO system in tumor growth, angiogenesis and metabolism - Targeting HO-1 as an anti-tumor therapy. Vascul Pharmacol 74: 11-22. doi: 10.1016/j. vph.2015.09.004

Mali P, Yang L, Esvelt KM, Aach J, Guell M, DiCarlo JE, Norville JE, Church GM (2013) RNA-guided human genome engineering via Cas9. Science 339: 823-826. doi: 10.1126/science.1232033

Metz R, Duhadaway JB, Rust S, Munn DH, Muller AJ, Mautino M, Prendergast GC (2010) Zinc protoporphyrin IX stimulates tumor immunity by disrupting the immunosuppressive enzyme indoleamine 2,3-dioxygenase. Mol Cancer Ther 9: 1864-1871. doi: 10.1158/15357163.MCT-10-0185

Ogawa K, Sun J, Taketani S, Nakajima O, Nishitani C, Sassa S, Hayashi N, Yamamoto M, Shibahara S, Fujita H, Igarashi K (2001) Heme mediates derepression of Maf recognition element through direct binding to transcription repressor Bach1. EMBO J 20: 28352843. doi: $10.1093 / \mathrm{emboj} / 20.11 .2835$

Peng R, Lin G, Li J (2016) Potential pitfalls of CRISPR/Cas9-mediated genome editing. FEBS I 283: 1218-1231. doi: 10.1111/febs.13586

Qiu S, Adema CM, Lane T (2005) A computational study of off-target effects of RNA interference. Nucleic Acids Res 33: 1834-1847. doi: $10.1093 /$ nar/gki324

Rahman MN, Vlahakis JZ, Vukomanovic D, Szarek WA, Nakatsu K, Jia Z (2009) X-ray crystal structure of human heme oxygenase-1 with $(2 \mathrm{R}, 4 \mathrm{~S})-2-[2-(4-$ chlorophenyl)ethyl $]-2-[(1 \mathrm{H}$-imidazol-1-yl) methyl]-4[((5-trifluorom ethylpyridin-2-yl)thio)methyl]-1,3-dioxolane: a novel, inducible binding mode. J Med Chem 52: 4946-4950. doi: $10.1021 /$ jm $900434 \mathrm{f}$

Rao DD, Vorhies JS, Senzer N, Nemunaitis J (2009) siRNA vs. shRNA: similarities and differences. Adv Drug Deliv Rev 61: 746-759. doi: 10.1016/j.addr.2009.04.004

Reynolds A, Anderson EM, Vermeulen A, Fedorov Y, Robinson K, Leake D, Karpilow J, Marshall WS, Khvorova A (2006) Induction of the interferon response by siRNA is cell type- and duplex lengthdependent. RNA 12: 988-993. doi: 10.1261/rna.2340906

Sass G, Leukel P, Schmitz V, Raskopf E, Ocker M, Neureiter D, Meissnitzer M, Tasika E, Tannapfel A, Tiegs G (2008) Inhibition of heme oxygenase 1 expression by small interfering RNA decreases orthotopic tumor growth in livers of mice. Int J Cancer 123: 12691277. doi: 10.1002 /ijc. 23695

Schulz S, Wong RJ, Vreman HJ, Stevenson DK (2012) Metalloporphyrins - an update. Front Pharmacol 3: 68. doi: 10.3389/ fphar.2012.00068

Stocker R (1990) Induction of haem oxygenase as a defence against oxidative stress. Free Radic Res Commun 9: 101-112 
Szulc J, Wiznerowicz M, Sauvain MO, Trono D, Aebischer P (2006) A versatile tool for conditional gene expression and knockdown. Nat Methods 3: 109-116. doi: 10.1038/nmeth846

Tenhunen R, Marver HS, Schmid R (1968) The enzymatic conversion of heme to bilirubin by microsomal heme oxygenase. Proc Natl Acad Sci U S A 61: 748-755

Wang S, Avery JE, Hannafon BN, Lind SE, Ding WQ (2013) Zinc protoporphyrin suppresses cancer cell viability through a heme oxygenase-1-independent mechanism: the involvement of the Wnt/be- ta-catenin signaling pathway. Biochem Pharmacol 85: 1611-1618. doi: 10.1016/j.bcp.2013.03.011

Xie K, Yang Y (2013) RNA-guided genome editing in plants using a CRISPR-Cas system. Mol Plant 6: 1975-1983. doi: 10.1093/mp/ sst119

Yang G, Nguyen X, Ou J, Rekulapelli P, Stevenson DK, Dennery PA (2001) Unique effects of zinc protoporphyrin on HO-1 induction and apoptosis. Blood 97: 1306-1313 